\title{
A Young Male With Arthritis and Skin Lesions
}

\author{
Vera HOVING (1)', Evert Jan Ter BORG (1) ${ }^{2}$ \\ ${ }^{1}$ Department of Internal Medicine, Sint Antonius Hospital, Nieuwegein, Netherlands \\ ${ }^{2}$ Department of Rheumatology, Sint Antonius Hospital, Nieuwegein, Netherlands
}

Dissemination of gonococci occurs rarely, but can cause specific syndromes, characterized by tenosynovitis, arthralgia, and skin lesions. A definite diagnosis may be difficult because disseminated gonococcal infection (DGI) is often associated with sterile cultures. In this article, we report a male patient with arthritis and skin lesions as first manifestations of a gonococcal infection.

A 20-year-old male patient presented to the Emergency Department because of a five-day history of swollen right wrist and erythematous skin lesions. After starting prednisone, the symptoms improved initially, but after a few days he got migrating joint pains. A few weeks earlier, he had a throat infection without fever. There was no history of any urogenital symptoms. On physical examination, his left wrist was tender on palpation and there was remarkable peripheral edema of his left hand (Figure 1). There was also mild arthritis of his right ankle. There were small, painless skin lesions with crusts on his both elbows and right tibia (Figure 2). Aspiration of the synovial fluid showed purulent fluid. Polymerase chain reaction and culture of this aspirate were positive for gonococci. Antibiotic treatment (ceftriaxone) was started. Arthroscopic lavage of the left wrist was performed because of persisting pains. Thereafter, his symptoms improved and inflammation parameters decreased.
Gonococci are transmitted by sexual contact, mainly through mucosal membranes. DGI is rare and occurs in $0.5-3.0 \%$ of patients infected with $N$. gonorrhoea. ${ }^{1}$ It generally occurs several days to two weeks after primary genital infection. Asymptomatic mucosal infection is thought to be the predisposing factor for dissemination in

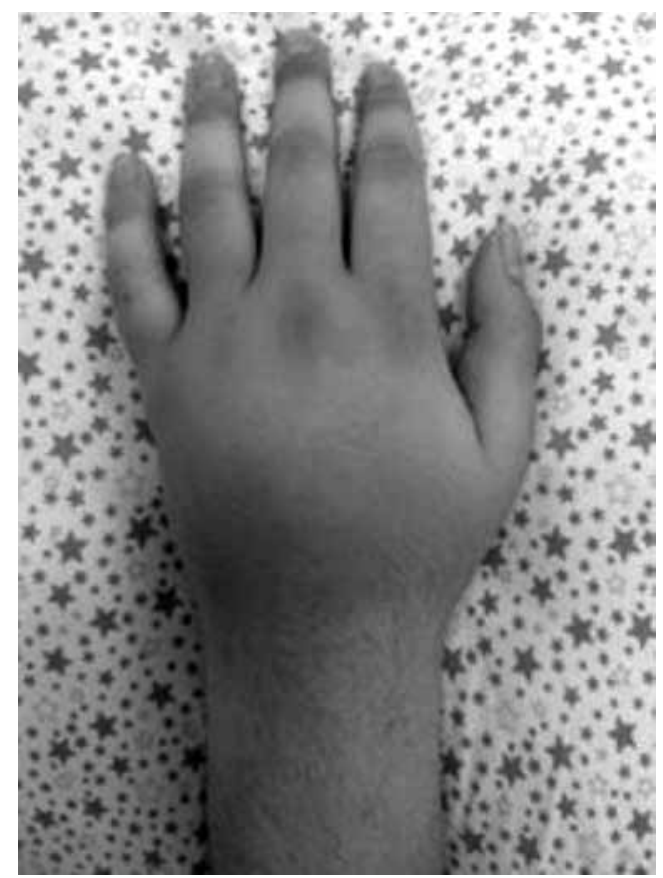

Figure 1. Arthritis of left wrist.

Received: December 21, 2017 Accepted: January 11, 2018 Published online: January 29, 2018 


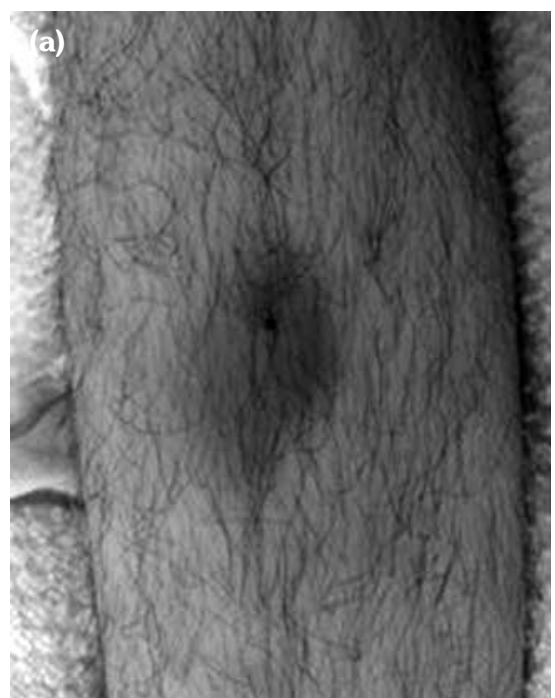

Figure 2. (a) Skin lesion of right tibia.

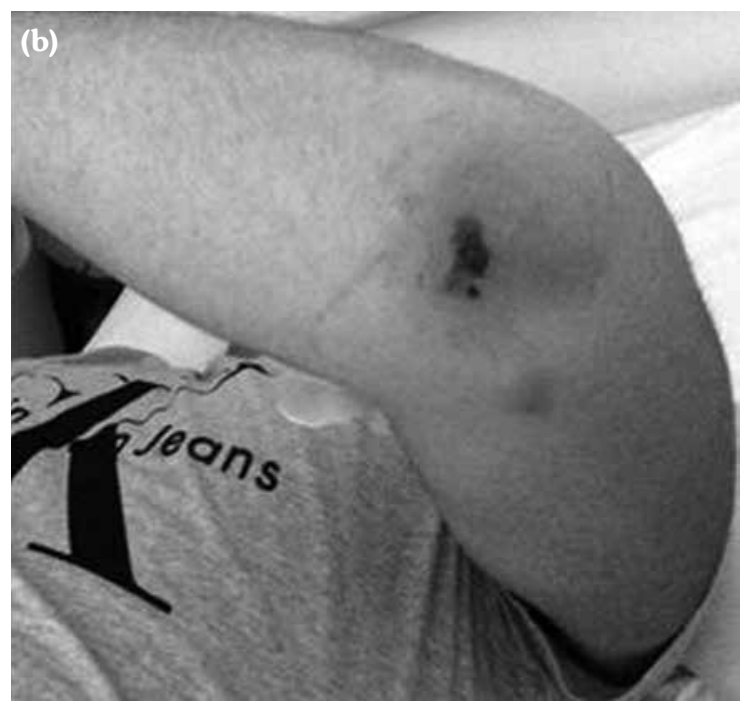

(b) Skin lesion of left elbow. most patients. $^{2,3}$ Strains causing DGI usually do not present with symptoms of urethral discharge, which was the case in our patient. ${ }^{4}$ The common manifestations of DGI are the "arthritis-dermatitis syndrome" and "purulent arthritis". The arthritis-dermatitis syndrome is characterized by the triad of tenosynovitis, dermatitis, and polyarthralgias without purulent arthritis. Fever and generalized malaise occur in $60 \%$ of the patients. Tenosynovitis is a unique finding. Skin lesions occur in $75 \%$ and consist of painless lesions, which are usually pustular or vesiculopustular. ${ }^{1}$ The purulent arthritis typically presents with an abrupt onset of arthritis. There are no skin lesions and most patients are afebrile. There are also patients with overlap of the two manifestations, like our patient. ${ }^{1,2}$ The diagnosis is established by identification of $N$. gonorrhoea on a specimen of blood, synovial fluid or skin lesion. Mostly, cultures of synovial fluid and skin lesions are negative, but positive blood cultures are found in $50 \%$ of patients with DGI that present with arthritis-dermatitis syndrome. In case of purulent arthritis, N. gonorrhoea is found in fewer than $50 \%$ of purulent synovial effusions. ${ }^{5}$ Intravenous ceftriaxone should be used to treat all cases of gonococcal infection. In case of DGI, patients should be treated for at least one week intravenously.

In conclusion, DGI is seen rarely and cultures are often negative, but it should be kept in mind in sexually active people who have features of the classic triad of tenosynovitis, dermatitis, and polyarthralgias-even when they have no genitourinary symptoms.

\section{Declaration of conflicting interests}

The authors declared no conflicts of interest with respect to the authorship and/or publication of this article.

\section{Funding}

The authors received no financial support for the research and/or authorship of this article.

\section{REFERENCES}

1. Goldenberg DL. Disseminated gonococcal infection. UpToDate. Available from: https:// www-uptodate-com.proxy-ub.rug.nl/contents/ disseminated-gonococcal-infection?source $=$ search $_{-}$ result\&search $=$ disseminated $\% 20$ gonococcal $\% 20$ infection\&selectedTitle $=1 \sim 45$.

2. Doesschate TT, Wismans PJ, Slobbe L. Een man met een gedissemineerde gonokokkeninfectie. Tijdschr Infect 2015;10:44-8.

3. Lohani S, Nazir S, Tachamo N, Patel N. Disseminated gonococcal infection: an unusual presentation. J Community Hosp Intern Med Perspect 2016;6:31841.

4. Jain S, Win HN, Chalam V, Yee L. Disseminated gonococcal infection presenting as vasculitis: a case report. J Clin Pathol 2007;60:90-1.

5. Suzaki A, Hayashi K, Kosuge K, Soma M, Hayakawa S. Disseminated gonococcal infection in Japan: a case report and literature review. Intern Med 2011;50:2039-43. 